\title{
PCMT1 gene polymorphisms, maternal folate metabolism, and neural tube defects: a case-control study in a population with relatively low folate intake
}

\author{
Fang Wang $\cdot$ Jianhua Wang $\cdot$ Jin Guo $\cdot$ Xiaoli Chen $\cdot$ \\ Zhen Guan • Huizhi Zhao $\cdot$ Hua Xie • Chi Liu • \\ Yihua Bao $\cdot$ Jizhen Zou $\cdot$ Bo Niu $\cdot$ Ting Zhang
}

Received: 16 February 2013/Accepted: 26 July 2013/Published online: 6 August 2013

(c) Springer-Verlag Berlin Heidelberg 2013

\begin{abstract}
The PCMT1 gene encodes the protein repair enzyme protein-L-isoaspartate (D-aspartate) O-methyltransferase, which is known to protect certain neural cells against Bax-induced apoptosis. Previous studies have produced inconsistent results regarding the effects of PCMTI (rs4816 and rs4552) polymorphisms on neural tube defects (NTDs). Reduced maternal plasma folate levels and/or elevated homocysteine (Hcy) levels are considered to be risk factors for NTDs. In order to clarify the key factors contributing to the apparent discrepancy and investigate gene-environment interaction, we conducted a case-control study including 121 cases and 146 matched controls to investigate the association between the two PCMT1 polymorphisms in fetuses and the risk of NTDs in the Chinese population of Lvliang, which has low folate intake. Maternal plasma folate and Hcy levels were also measured, and the interaction between fetal PCMTI gene status and maternal folate metabolites was assessed. Maternal plasma folate concentrations in the NTD group were lower than in controls (10.23 vs. $13.08 \mathrm{nmol} / \mathrm{L}$, adjusted $P=0.059)$, and
\end{abstract}

Fang Wang and Jianhua Wang have contributed equally to this manuscript.

F. Wang $\cdot$ J. Wang $\cdot$ J. Guo $\cdot$ X. Chen $\cdot$ Z. Guan $\cdot$ H. Zhao ·

H. Xie · C. Liu - Y. Bao · B. Niu · T. Zhang $(\bowtie)$

Beijing Municipal Key Laboratory of Child Development

and Nutriomics, Capital Institute of Pediatrics,

Beijing 100020, China

e-mail: zhangtingcv@yahoo.com.cn

J. Zou

Department of Pathology, Capital Institute of Pediatrics,

Beijing 100020, China
Hcy concentrations were significantly higher (14.46 vs. $11.65 \mu \mathrm{mol} / \mathrm{L}$, adjusted $P=0.026$ ). Fetuses carrying the rs4816 AG + GG genotype, combined with higher maternal plasma Hcy, had a 6.46-fold $(95 \%$ CI 1.15-36.46) increased risk of anencephaly. The results of this study imply that the fetal PCMT1 rs4816 polymorphism may play only a weak role in NTD formation and that gene-environment interactions might be more significant.

Keywords Association study - PCMT1 .

Homocysteine $\cdot$ Neural tube defect .

Gene-environment interaction

\section{Introduction}

Neural tube defects (NTDs) are relatively common, severe congenital malformations caused by multiple genes and environmental factors. NTDs include a range of malformations (e.g., spina bifida, anencephaly), which further complicates the identification of risk factors. Periconceptional folic acid supplementation has been reported to prevent 50-70\% of NTDs (Czeizel and Dudas 1992; De Wals et al. 2007). We previously demonstrated that nutrient deficiency is an important independent risk factor for birth defects (Zhang et al. 2008a), and lower plasma concentrations of folate and vitamin $\mathrm{B} 12$ are related to an increased risk of NTDs in high-risk populations (Zhang et al. 2009). Many polymorphisms in folate pathway genes have been investigated and shown to be associated with NTD risk (Zhu et al. 2003; van der Linden et al. 2006; Boyles et al. 2006). However, none of the known folate pathway gene variants contribute substantially to the population burden of NTDs. 
The human PCMTI gene encodes the protein repair enzyme protein-L-isoaspartate (D-aspartate) O-methyltransferase (PIMT), which initiates the conversion of abnormal D-aspartyl and L-isoaspartyl residues to the normal L-aspartyl form by transferring a methyl group from S-adenosyl-L-methionine (SAM) to the free carboxyl groups of D-aspartyl and L-isoaspartyl residues. PIMT is one of dozens of methyltransferases involved in the onecarbon compound metabolic process and a crucial factor for the posttranslational modification of proteins. In mammals, PIMT is expressed in all tissues, with the highest levels found in the brain (Kim et al. 1997). PIMT is known to protect certain neural cells from Bax-induced apoptosis through an SAM-binding motif-dependent mechanism (Huebscher et al. 1999) and to repair abnormal proteins to maintain normal mitogen-activated protein kinase (MAPK) signaling (Kosugi et al. 2008). Pcmt1 null (pcmtl(-/-)) mice present with significantly higher levels of SAM and lower levels of S-adenosylhomocysteine (SAH) in their brains compared with their wild-type littermates (Farrar and Clarke 2002). They accumulate Lisoaspartyl residues in several tissues and die, on average, 42 days after birth from progressive epileptic seizures (Kim et al. 1997). The Pcmt1 gene was upregulated in Folr1 (folate-binding protein 1) nullizygous mouse neural tube tissues when the normal phenotype was rescued with folic acid supplementation (Spiegelstein et al. 2004). Therefore, PIMT might act to trigger the repair of isoaspartate protein damage, and this damage might increase if Hcy or SAH levels are elevated or folate levels are reduced. PCMT1 is thus an attractive candidate for screening for NTD risk.

Zhu et al. reported that PCMT1 gene polymorphisms (rs4816 and rs4552) in infants were a genetic modifier for the risk of spina bifida in a Californian population (Zhu et al. 2006). However, our recent research showed that maternal rs4816 polymorphism might be a potential genetic risk factor for anencephaly, but not for spina bifida (Zhao et al. 2012). This result did not fully support the findings of Zhu et al. and it is possible that the differences between the study populations (ethnic group, fetus/mother, with or without folic supplementation) may have been the key factors contributing to this apparent discrepancy. Reduced maternal plasma folate levels and/ or elevated Hcy levels were considered to be risk factors for NTDs (Felkner et al. 2009). In order to clarify the key factors contributing to this apparent discrepancy and investigate possible gene-environment interactions, we analyzed the association between fetal PCMT1 polymorphisms and NTDs and explored the interaction between fetal PCMTI genotype and maternal environmental factors in this study.

\section{Methods}

Subjects

The study was conducted in the Lvliang mountain area of Shanxi Province in Northern China, which was shown to have an NTD prevalence of 199.38 per 10,000 in our recently completed survey (Gu et al. 2007). Previous studies found that women of childbearing age in this region had a remarkably insufficient intake of some nutrients, especially folic acid (Zhang et al. 2008a).

Stillborn NTD cases were obtained from nine county hospitals in the area from 2004 to 2007. Cases were medical abortions diagnosed with NTDs by B-mode ultrasound, in which the sex, gestational age, and general development of the fetus were recorded in detail. A pathologic diagnosis of NTD was performed by experienced pathologists according to the International Classification of Diseases, Tenth Revision, codes Q00 anencephaly, Q05 spina bifida, and Q01 encephalocele. Control fetuses that had been aborted for non-medical reasons were enrolled from the same region. Control fetuses were matched with NTD cases based upon maternal age and number of gestational weeks. Any fetuses displaying pathologic malformations or intrauterine growth retardation were excluded from the control group.

Routine prenatal checkups, questionnaire interviews, and autopsies were completed for both control and case subjects. Fasting blood samples were collected from pregnant women just before abortion and were immediately centrifuged at $2,500 \mathrm{rpm}$ for $10 \mathrm{~min}$. Autopsy samples for DNA extraction and separated plasma were stored in local hospitals at $-20{ }^{\circ} \mathrm{C}$ before shipping on ice to the laboratories in Beijing. Neural tissues (brain tissue and residues of brain tissue) were obtained from case and control subjects. The study was approved by the local ethics committee, and written informed consent was obtained from the parents of all fetuses.

\section{DNA extraction}

Genomic DNA was extracted from frozen tissue samples using the Blood and Tissue DNA Kit (Qiagen, Germany) according to the manufacturer's instructions and was subsequently used for genotyping. The concentration and purity of the DNA were determined by absorbance at 260 and $280 \mathrm{~nm}$.

Biochemical measures

We followed the protocol described in our previous study (Wang et al. 2010) to detect maternal plasma 
concentrations of folate and Hcy. Briefly, plasma folate was measured using a competitive receptor-binding immunoassay (Access II; Beckman Coulter, Krefeld, Germany). Hcy was measured using a Hitachi Model 7170A automatic analyzer (Hitachi, Tokyo, Japan) with a Hcy assay kit (Jiu-Qiang Company, Beijing, China). The intraassay coefficients of variability for folate and Hcy were $3.8-6.5 \%$ and $2.6-4.0 \%$, respectively. We selected 44 cases and 44 controls with larger volumes of plasma for detection. Only three cases and four controls had taken periconceptional folic acid supplements. The population demographics of these selected subjects were similar to those of the original groups.

\section{Genotyping}

PCMT1 polymorphisms (rs4552 and rs4816) were genotyped using high-resolution DNA melting (HRM) analysis (LightScanner96, Idaho Technology Inc.). The primers and probes were as described in our previous study (Zhao et al. 2012). To ensure genotyping consistency, $10 \%$ of samples were re-genotyped, and $10 \%$ of samples were sequenced directly to validate the accuracy of the genotyping. Sequencing results were exported to Mutation Surveyor version 3.25 (Softgenetics, State College, PA, USA; http:// www.softgenetics.com) for alignment and multiple comparisons.

\section{Statistical analysis}

The lifestyle and sociodemographic characteristics of the patients were compared using $\chi^{2}$ tests for categorical variables. The Hardy-Weinberg equilibrium (HWE) was also tested using $\chi^{2}$ tests, and $P>0.05$ was considered to indicate that the distribution of genotypes did not deviate from expected HWE frequencies. Differences in PCMTI genotype/allele frequencies between case and control groups were tested using $\chi^{2}$ or Fisher's exact tests. Odds ratios (ORs) and $95 \%$ confidence intervals (CIs) were calculated to estimate the risks of NTDs related to the polymorphisms. Plasma folate and Hcy concentrations were skewed and were normalized by logarithmic transformation for all analyses. We combined our data with that from our previous study (Zhao et al. 2012) to assess the interaction between maternal and fetal genotypes using a logistic regression model. A linear regression model was used to compare log-transformed compound concentrations between the groups and adjust for age, educational level, gravidity, folate supplementation, gestational week, and fetal sex. In addition, ORs (95\% CIs) for genotypes in NTD vs. control cases stratified by NTD subtypes and by maternal plasma folate and Hcy levels were calculated using $\chi^{2}$ tests. Analyses were performed using the SPSS
11.5 software package (McGraw-Hill Inc, New York, NY, USA). All $P$ values were two-sided, and $P<0.05$ was considered to be significant. The linkage disequilibrium and haplotype association between rs4816 and rs4552 with NTDs were estimated using Haploview (http://www.broad. mit.edu/mpg/haploview/) (Barrett et al. 2005).

\section{Results}

We recruited 124 cases and 151 matched controls. Among the NTD subjects, two declined to participate and one case whose NTD phenotype was not confirmed by autopsy was excluded, giving a participation rate of $97.6 \%$. Among the matched control subjects, three declined to participate and two were excluded because of pathological malformations or intrauterine growth retardation detected at autopsy, giving a control participation rate of $96.7 \%$. The characteristics of the participating subjects are shown in Table 1. The distributions of maternal age, educational background, gravidity, periconceptional folic acid used, and fetus sex

Table 1 Epidemiologic characteristics of NTD and control groups

\begin{tabular}{|c|c|c|c|}
\hline Characteristics & $\begin{array}{l}\text { NTDs } \\
n\left(\%^{\mathrm{a}}\right)\end{array}$ & $\begin{array}{l}\text { Control } \\
n\left(\%^{\mathrm{a}}\right)\end{array}$ & $P^{\mathrm{c}}$ \\
\hline & $121^{\mathrm{b}}$ & $146^{\mathrm{b}}$ & \\
\hline Mother's age (years) & 121 & 145 & 0.131 \\
\hline$<20$ & $11(9.1)$ & $14(9.7)$ & \\
\hline $20-24$ & $45(37.2)$ & $37(25.5)$ & \\
\hline $25-29$ & $42(34.7)$ & $52(35.9)$ & \\
\hline$\geq 30$ & $23(19.0)$ & $42(29.0)$ & \\
\hline Mother's education & 114 & 141 & 0.792 \\
\hline$\geq$ High school graduation & $18(15.8)$ & $24(17.0)$ & \\
\hline Gravidity $(n)$ & 114 & 141 & 0.086 \\
\hline 1 & $54(47.4)$ & $50(35.5)$ & \\
\hline 2 & $41(36.0)$ & $54(38.3)$ & \\
\hline$\geq 3$ & $19(16.7)$ & $37(26.2)$ & \\
\hline $\begin{array}{l}\text { Periconceptional folic acid } \\
\text { use }^{\mathrm{d}}\end{array}$ & 121 & 146 & 0.570 \\
\hline Yes & $7(5.8)$ & $11(7.5)$ & \\
\hline Gestational age (weeks) & 90 & 120 & $<0.001$ \\
\hline$<18$ & $20(22.2)$ & $35(29.2)$ & \\
\hline $18-24$ & $44(48.9)$ & $79(65.8)$ & \\
\hline$>24$ & $26(28.9)$ & $6(5.0)$ & \\
\hline Fetus sex & 121 & 144 & 0.937 \\
\hline Male & $49(40.5)$ & $59(41.0)$ & \\
\hline
\end{tabular}

\footnotetext{
${ }^{a}$ Percentages may not equal 100 because of rounding

b Number of subjects

c $\chi^{2}$ test was used to calculate $P$ values

d Periconceptional refers to the month before conception and the first 3 months after conception
} 
were similar between the case and control groups. The mean gestational week of controls was lower than that for cases, because some pregnant women underwent abortions during early pregnancy for non-medical reasons. The level of periconceptional folic acid usage was low in both groups: $5.8 \%$ in the NTD group and $7.5 \%$ in the control group.

The two polymorphisms were successfully genotyped using HRM; rs4816 was detected in 116 (95.9\%) NTD cases and 141(96.6\%) controls, and rs4552 in 120 $(99.2 \%)$ NTD cases and $144(98.6 \%)$ controls. The reproducibility of genotyping was high, with $100 \%$ concordance between the HRM and direct sequencing genotyping methods. Both polymorphisms were in HWE among the NTD and control groups (data not shown), and the genotype distributions showed no significant differences between the NTD and control groups (Table 2). For linkage between the two SNPs $\left(D^{\prime}=0.96, r^{2}=0.77\right)$, we focused on the non-synonymous SNP rs4816 for analysis. No association was found between rs4816 and NTD subtypes (Table 3).

Measurement of maternal plasma folate and Hcy using a competitive receptor-binding immunoassay and Hcy assay kit demonstrated a significantly higher level of Hcy $(14.46 \pm 7.44 \mu \mathrm{mol} / \mathrm{L})$ in the NTD group compared with the control group $(11.65 \pm 6.23 \mu \mathrm{mol} / \mathrm{L}, \quad$ adjusted $P=0.026$ ), while the difference in plasma folate levels between the NTD and control groups was of borderline significance (adjusted $P=0.059$ ). The results were similar to those in previous reports from our group (Wang et al. 2010).

Table 4 presents the ORs for PCMT1 rs4816 genotype in NTD fetuses versus controls stratified according to maternal plasma folate and Hcy levels. Maternal plasma folate results were dichotomized, with a cut-off point at the 25th percentile $(55.00 \mathrm{pmol} / \mathrm{L})$, and the Hcy results were dichotomized with a cut-off point at the 75th percentile $(16.00 \mu \mathrm{mol} / \mathrm{L})$ in all NTDs and controls. Fetuses carrying the $\mathrm{AG}+\mathrm{GG}$ genotype in combination with higher maternal plasma concentrations of Hcy had a 6.46-fold (95\% CI 1.15-36.46) increased risk of anencephaly. Maternal folate concentration was not associated with risk. The risk of encephalocele was not studied because there were too few samples (11 samples).

We merged this data set with our previous data (Zhao et al. 2012) and analyzed a combined 113 mother-fetus pairs (67 control pairs and 46 case pairs). These combined results showed that the interaction between maternal and fetal genotypes was not significant.

\section{Discussion}

NTDs are a group of heterogeneous and complex congenital abnormalities, the phenotypic severity of which varies with the type and level of the lesion (Finnell et al. 2003). NTDs are now the leading cause of infant mortality and inflict a large economic burden on society and families

Table 2 Genotype distributions and allele frequencies of PCMT1 rs4816 and rs4552 in NTD and control groups

\begin{tabular}{|c|c|c|c|c|c|}
\hline Polymorphism & Genotype & NTDs $n(\%)$ & Control $n(\%)$ & OR $(95 \% \mathrm{CI})$ & $P^{\mathrm{a}}$ \\
\hline \multirow[t]{4}{*}{ rs4816: A > G (Ile120Val) } & & 116 & 141 & & \\
\hline & AA & $70(60.3)$ & $87(61.7)$ & 1 & \\
\hline & AG & 37 (31.9) & $45(31.9)$ & $1.02(0.597,1.748)$ & 0.937 \\
\hline & GG & $9(7.8)$ & $9(6.4)$ & $1.24(0.468,3.299)$ & 0.191 \\
\hline \multirow[t]{4}{*}{ rs 4552: $\mathrm{T}>\mathrm{A}$} & & 120 & 144 & & \\
\hline & $\mathrm{TT}$ & $75(62.5)$ & $91(63.2)$ & 1 & \\
\hline & AT & $42(35.0)$ & $48(33.3)$ & $1.06(0.635,1.776)$ & 0.820 \\
\hline & AA & $3(2.5)$ & $5(3.5)$ & $0.73(0.168,3.146)$ & 0.670 \\
\hline
\end{tabular}

${ }^{\mathrm{a}} \chi^{2}$ test was used to calculate $P$ values

Table 3 Distribution of rs4816 and association with NTD subtypes

\begin{tabular}{lllll}
\hline Genotypes & Control $(n=141), n(\%)$ & Anencephaly $(n=50), n(\%)$ & Spina bifida $(n=55), n(\%)$ & Encephalocele $(n=11), n(\%)$ \\
\hline AA & $87(61.7)$ & $29(58.0)$ & $36(65.5)$ & $5(45.5)$ \\
AG/GG & $54(38.3)$ & $21(42.0)$ & $19(34.5)$ & $6(54.5)$ \\
OR $(95 \% \mathrm{CI})$ & & $1.17(0.61,2.25)$ & $0.85(0.44,1.63)$ & $1.93(0.56,6.64)$ \\
$P^{\mathrm{a}}$ & & 0.645 & 0.625 & 0.288
\end{tabular}

${ }^{\mathrm{a}} \chi^{2}$ test was used to calculate $P$ values 
Table 4 Calculated odds ratios (95\% confidence interval) for rs4816 A > G genotypes in NTD versus control cases, classified by NTD subtype and by maternal plasma folate and Hcy levels

\begin{tabular}{|c|c|c|c|c|c|c|}
\hline & \multicolumn{2}{|c|}{ All NTDs } & \multicolumn{2}{|c|}{ Spina bifida } & \multicolumn{2}{|c|}{ Anencephaly } \\
\hline & $n$ & OR $(95 \% \mathrm{CI})$ & $n$ & OR $(95 \% \mathrm{CI})$ & $n$ & OR $(95 \%$ CI $)$ \\
\hline \multicolumn{7}{|c|}{ Folate low $(<25 \text { th })^{\text {ac }}$} \\
\hline $\mathrm{rs} 4816 \mathrm{AG} / \mathrm{GG}$ & 5 & $2.3(0.56-9.04)$ & 2 & $1.8(0.26-12.41)$ & 3 & $4.1(0.59-27.92)$ \\
\hline rs4816 AA & 6 & Referent & 3 & Referent & 2 & Referent \\
\hline \multicolumn{7}{|c|}{ Folate high $(\geq 25 \text { th })^{\text {ac }}$} \\
\hline $\mathrm{rs} 4816 \mathrm{AG} / \mathrm{GG}$ & 10 & $2.3(0.74-6.82)$ & 2 & $1.1(0.18-6.49)$ & 6 & $2.3(0.63-8.58)$ \\
\hline rs4816 AA & 12 & Referent & 5 & Referent & 7 & Referent \\
\hline \multicolumn{7}{|l|}{ Hcy low $(\leq 75 \text { th })^{\text {bd }}$} \\
\hline rs4816 AG/GG & 13 & $2.0(0.74-5.44)$ & 6 & $1.8(0.52-6.60)$ & 5 & $1.5(0.41-5.78)$ \\
\hline rs4816 AA & 14 & Referent & 7 & Referent & 7 & Referent \\
\hline \multicolumn{7}{|c|}{ Hcy high $(>75 \text { th })^{\text {bd }}$} \\
\hline rs4816 AG/GG & 6 & $\begin{array}{l}2.6 \\
\quad(0.67-10.04)\end{array}$ & 0 & - & 6 & $\begin{array}{l}6.46 \\
\quad(1.15-36.46)\end{array}$ \\
\hline rs4816 AA & 5 & Referent & 2 & Referent & 2 & Referent \\
\hline
\end{tabular}

(Davidoff et al. 2002). It has been reported that NTDs can be prevented with periconceptional folic acid supplementation (Czeizel and Dudas 1992; De Wals et al. 2007). Actions have been taken by the Chinese Ministry of Health to reduce the incidence of NTDs in China since 2009. Free folic acid supplementation is offered to rural women who are preparing for pregnancy. We analyzed 267 DNA samples from a Chinese population in the Lvliang area, which has the highest rate of NTDs in China, more than 10 times higher than in most epidemiological studies performed in other parts of the world. Impairments in folateand homocysteine-mediated one-carbon metabolism are associated with NTDs in this region (Zhang et al. 2008b). This study therefore concentrated on investigating the relationships among PCMT1 gene polymorphisms, maternal folate metabolism, and NTDs.

We found no significant association between fetal PCMT1 polymorphisms and total NTDs, or any of the three NTD subtypes. For linkage between the two SNPs $\left(D^{\prime}=0.96, r^{2}=0.77\right)$, we focused on the non-synonymous SNP rs4816. Fetuses carrying the rs 4816 AG + GG, combined with the higher maternal plasma Hcy levels, had a 6.46-fold increased risk of anencephaly. This result conflicts with the conclusions with Zhu et al.'s study, which found that the PCMT1 rs4816 polymorphism was a genetic modifier for the risk of spina bifida in a Californian population. This apparent discrepancy for the risk of spina bifida may be due to differences in study subjects, sample sizes and/or ethnicity between the studies. It has been reported that the degree of association between methylenetetrahydrofolate reductase (MTHFR) polymorphisms in the folate pathway and NTDs varies according to ethnicity and region (Relton et al. 2003; Boduroglu et al. 1998; Botto and Yang 2000). The findings of the current study suggest that the importance of different polymorphisms in terms of specific NTD phenotypes might vary depending on ethnicity and region. This study was conducted within a relatively small sample size, and a larger sample size may be required to more completely reveal the relationships among genotype, environment, and NTDs.

Previous experimental studies on mouse NTD models and analysis of inheritance patterns in humans suggest that NTDs result from the interplay of multiple genes, as well as interactions between gene and environment, rather than being monogenic malformations (Harris and Juriloff 2007; Holmes et al. 1976; Shaw et al. 1994; van der Put et al. 2001). Among the potential environmental factors, maternal folate status is known to be a protective factor against NTDs. Some mothers who have babies with NTDs have reduced plasma folate levels and/or elevated Hcy levels. 
Therefore, low plasma folate and/or high Hcy was considered to be risk factors for NTDs (Felkner et al. 2009). In the current study, it was demonstrated that the rs4816 AG + GG genotype only increased the risk of anencephaly for mothers with higher Hcy levels, and it was not associated with the occurrence of spina bifida, though these results might have been influenced by phenotypic heterogeneity and the small sample size. Anencephaly is potentially a more severe disease than spina bifida and encephalocele.

Hcy level in plasma is a sensitive indicator for folate deficiencies (Allen et al. 1993). High Hcy is related to pregnancy complications (Steegers-Theunissen et al. 1992) and NTDs (Steegers-Theunissen et al. 1994; Rosenquist et al. 1996). Hcy is a metabolite of the essential amino acid methionine and exists at a critical biochemical intersection in the methionine metabolic pathway. SAM is produced from methionine by adding adenosine triphosphate (ATP). SAM is converted to SAH by donating a methyl via methyltransferase. Adenosylhomocysteinase converts SAH to Hcy, which is re-methylated by 5-methyltetrahydrofolate back to methionine. It is reported that changes in the SAM/SAH ratio affected liver PIMT activity in rats after betaine administration (Kharbanda et al. 2007, 2009; Ghandour et al. 2002; Carter and Aswad 2008). Moreover, SAH is a potent inhibitor of many methyltransferases including PIMT. It is possible that PIMT acts to trigger the repair of isoaspartate protein damage and that this damage increases as a result of elevation of SAH levels or folate reduction. In previous reports from our group, we observed significantly lower plasma concentrations of folate and remarkably higher concentrations of SAH in cases than in controls (Zhang et al. 2008b). However, our maternal plasma quantity did not reach the minimum required for detecting SAH levels.

The functional polymorphism in rs4816 results in an Ile120Val change in the PIMT protein, thus affecting the basal level of enzyme activity. The rs $4816 \mathrm{G}$ allele encodes a protein isoform with low enzymatic activity and thermostability, but high SAM affinity in red blood cells, which might affect the methyltransferase process (DeVry and Clarke 1999; David et al. 1997). The heterozygosity of $\mathrm{Val} / \mathrm{Il}$ favors efficient isoaspartate repair in vitro dynamics simulation studies (Rutherford and Daggett 2009). Our study showed that there was an increased anencephaly risk when the Val120 polymorphism with lowered protein repair activity combined with the presence of high Hcy. This might reduce the SAM/SAH ratio and PIMT activity. Ultimately, PIMT activity and substrate binding (influenced by the genotype) might influence repair of many proteins. It might become a risk factor if another prominent risk factor. The prominent risk factor further exacerbates the reduction in PIMT repair system activity and also impinges on the activities of other methyltransferases. Larger cohort studies are needed to further investigate the interplay between the biochemical metabolism and PCMT1 genotype.

In summary, this study explored the possible effects of fetal PCMT1 polymorphisms on the risk of NTD subtypes. The results suggest that the PCMT1 rs4816 polymorphism in fetuses may play only a weak role in NTD formation and that gene-environment interactions may be more important in the studied area where there is relatively low folate intake and a high incidence of NTDs.

Acknowledgments The authors disclosed receipt of the following financial support for the research and/or authorship of this article: the Ministry of Science and Technology of the P. R. China, National "973" project on Population and Health (2013CB945404), National Natural Science Foundation of China (Project 81070491). We are grateful to all the participants in this study and to all the obstetricians in the local hospital at Shanxi Province, as well as the pathologists in the Department of Pathology for the diagnoses. We also thank all the subjects and their families for their cooperation in providing both clinical information and samples for the study.

Conflict of interest The authors declare no conflict of interests.

\section{References}

Allen RH, Stabler SP, Savage DG, Lindenbaum J (1993) Metabolic abnormalities in cobalamin (vitamin B12) and folate deficiency. FASEB J 7(14):1344-1353

Barrett JC, Fry B, Maller J, Daly MJ (2005) Haploview: analysis and visualization of $\mathrm{LD}$ and haplotype maps. Bioinformatics 21(2):263-265. doi:10.1093/bioinformatics/bth457

Boduroglu K, Alikasifoglu M, Anar B, Tuncbilek E (1998) 677$>$ CT mutation on the methylenetetrahydrofolate reductase gene is not a risk factor for neural tube defects in Turkey. Arch Dis Child Fetal Neonatal Ed 78(3):F235

Botto LD, Yang Q (2000) 5,10-Methylenetetrahydrofolate reductase gene variants and congenital anomalies: a HuGE review. Am J Epidemiol 151(9):862-877

Boyles AL, Billups AV, Deak KL, Siegel DG, Mehltretter L, Slifer SH, Bassuk AG, Kessler JA, Reed MC, Nijhout HF, George TM, Enterline DS, Gilbert JR, Speer MC (2006) Neural tube defects and folate pathway genes: family-based association tests of gene-gene and gene-environment interactions. Environ Health Perspect 114(10):1547-1552

Carter WG, Aswad DW (2008) Formation, localization, and repair of L-isoaspartyl sites in histones $\mathrm{H} 2 \mathrm{~A}$ and $\mathrm{H} 2 \mathrm{~B}$ in nucleosomes from rat liver and chicken erythrocytes. Biochemistry 47(40):10757-10764. doi:10.1021/bi8013467

Czeizel AE, Dudas I (1992) Prevention of the first occurrence of neural-tube defects by periconceptional vitamin supplementation. N Engl J Med 327(26):1832-1835. doi:10.1056/ NEJM199212243272602

David CL, Szumlanski CL, DeVry CG, Park-Hah JO, Clarke S, Weinshilboum RM, Aswad DW (1997) Human erythrocyte protein L-isoaspartyl methyltransferase: heritability of basal activity and genetic polymorphism for thermal stability. Arch Biochem Biophys 346(2):277-286. doi:10.1006/abbi.1997.0303 
Davidoff MJ, Petrini J, Damus K, Russell RB, Mattison D (2002) Neural tube defect-specific infant mortality in the United States. Teratology 66(Suppl 1):S17-S22. doi:10.1002/tera.90005

De Wals P, Tairou F, Van Allen MI, Uh SH, Lowry RB, Sibbald B, Evans JA, Van den Hof MC, Zimmer P, Crowley M, Fernandez B, Lee NS, Niyonsenga T (2007) Reduction in neural-tube defects after folic acid fortification in Canada. N Engl J Med 357(2):135-142. doi:10.1056/NEJMoa067103

DeVry CG, Clarke S (1999) Polymorphic forms of the protein Lisoaspartate (D-aspartate) O-methyltransferase involved in the repair of age-damaged proteins. J Hum Genet 44(5):275-288. doi: $10.1007 / \mathrm{s} 100380050161$

Farrar C, Clarke S (2002) Altered levels of S-adenosylmethionine and S-adenosylhomocysteine in the brains of L-isoaspartyl (DAspartyl) O-methyltransferase-deficient mice. J Biol Chem 277(31):27856-27863. doi:10.1074/jbc.M203911200

Felkner M, Suarez L, Canfield MA, Brender JD, Sun Q (2009) Maternal serum homocysteine and risk for neural tube defects in a Texas-Mexico border population. Birth Defects Res A Clin Mol Teratol 85(6):574-581. doi:10.1002/bdra.20545

Finnell RH, Gould A, Spiegelstein O (2003) Pathobiology and genetics of neural tube defects. Epilepsia 44(Suppl 3):14-23

Ghandour H, Lin BF, Choi SW, Mason JB, Selhub J (2002) Folate status and age affect the accumulation of L-isoaspartyl residues in rat liver proteins. J Nutr 132(6):1357-1360

Gu X, Lin L, Zheng X, Zhang T, Song X, Wang J, Li X, Li P, Chen G, Wu J, Wu L, Liu J (2007) High prevalence of NTDs in Shanxi Province: a combined epidemiological approach. Birth Defects Res A Clin Mol Teratol 79(10):702-707. doi:10.1002/bdra. 20397

Harris MJ, Juriloff DM (2007) Mouse mutants with neural tube closure defects and their role in understanding human neural tube defects. Birth Defects Res A Clin Mol Teratol 79(3):187-210. doi:10.1002/bdra.20333

Holmes LB, Driscoll SG, Atkins L (1976) Etiologic heterogeneity of neural-tube defects. N Engl J Med 294(7):365-369. doi:10.1056/ NEJM197602122940704

Huebscher KJ, Lee J, Rovelli G, Ludin B, Matus A, Stauffer D, Furst P (1999) Protein isoaspartyl methyltransferase protects from Bax-induced apoptosis. Gene 240(2):333-341

Kharbanda KK, Mailliard ME, Baldwin CR, Sorrell MF, Tuma DJ (2007) Accumulation of proteins bearing atypical isoaspartyl residues in livers of alcohol-fed rats is prevented by betaine administration: effects on protein-L-isoaspartyl methyltransferase activity. J Hepatol 46(6):1119-1125. doi:10.1016/j.jhep. 2007.01.026

Kharbanda KK, Vigneswara V, McVicker BL, Newlaczyl AU, Bailey K, Tuma D, Ray DE, Carter WG (2009) Proteomics reveal a concerted upregulation of methionine metabolic pathway enzymes, and downregulation of carbonic anhydrase-III, in betaine supplemented ethanol-fed rats. Biochem Biophys Res Commun 381(4):523-527. doi:10.1016/j.bbrc.2009.02.082

Kim E, Lowenson JD, MacLaren DC, Clarke S, Young SG (1997) Deficiency of a protein-repair enzyme results in the accumulation of altered proteins, retardation of growth, and fatal seizures in mice. Proc Natl Acad Sci USA 94(12):6132-6137

Kosugi S, Furuchi T, Katane M, Sekine M, Shirasawa T, Homma H (2008) Suppression of protein L-isoaspartyl (D-aspartyl) methyltransferase results in hyperactivation of EGF-stimulated MEKERK signaling in cultured mammalian cells. Biochem Biophys Res Commun 371(1):22-27. doi:10.1016/j.bbrc.2008.03.109

Relton CL, Wilding CS, Jonas PA, Lynch SA, Tawn EJ, Burn J (2003) Genetic susceptibility to neural tube defect pregnancy varies with offspring phenotype. Clin Genet 64(5):424-428
Rosenquist TH, Ratashak SA, Selhub J (1996) Homocysteine induces congenital defects of the heart and neural tube: effect of folic acid. Proc Natl Acad Sci USA 93(26):15227-15232

Rutherford K, Daggett V (2009) The V119I polymorphism in protein L-isoaspartate O-methyltransferase alters the substrate-binding interface. Protein Eng Des Sel. 22(12):713-721. doi:10.1093/ protein/gzp056

Shaw GM, Jensvold NG, Wasserman CR, Lammer EJ (1994) Epidemiologic characteristics of phenotypically distinct neural tube defects among 0.7 million California births, 1983-1987. Teratology 49(2):143-149. doi:10.1002/tera.1420490210

Spiegelstein O, Cabrera RM, Bozinov D, Wlodarczyk B, Finnell RH (2004) Folate-regulated changes in gene expression in the anterior neural tube of folate binding protein-1 (Folbp1)deficient murine embryos. Neurochem Res 29(6):1105-1112

Steegers-Theunissen RP, Boers GH, Blom HJ, Trijbels FJ, Eskes TK (1992) Hyperhomocysteinaemia and recurrent spontaneous abortion or abruptio placentae. Lancet 339(8801):1122-1123

Steegers-Theunissen RP, Boers GH, Trijbels FJ, Finkelstein JD, Blom HJ, Thomas CM, Borm GF, Wouters MG, Eskes TK (1994) Maternal hyperhomocysteinemia: a risk factor for neural-tube defects? Metabolism 43(12):1475-1480

van der Linden IJ, Afman LA, Heil SG, Blom HJ (2006) Genetic variation in genes of folate metabolism and neural-tube defect risk. Proc Nutr Soc 65(2):204-215

van der Put NM, van Straaten HW, Trijbels FJ, Blom HJ (2001) Folate, homocysteine and neural tube defects: an overview. Exp Biol Med (Maywood) 226(4):243-270

Wang L, Wang F, Guan J, Le J, Wu L, Zou J, Zhao H, Pei L, Zheng X, Zhang T (2010) Relation between hypomethylation of long interspersed nucleotide elements and risk of neural tube defects. Am J Clin Nutr 91(5):1359-1367. doi:10.3945/ajcn.2009.28858

Zhang BY, Zhang T, Lin LM, Wang F, Xin RL, Gu X, He YN, Yu DM, Li PZ, Zhang QS, Zhao J, Qin YF, Yang XF, Chen G, Liu JF, Song XM, Zheng XY (2008a) Correlation between birth defects and dietary nutrition status in a high incidence area of China. Biomed Environ Sci 21(1):37-44. doi:10.1016/S08953988(08)60005-7

Zhang HY, Luo GA, Liang QL, Wang Y, Yang HH, Wang YM, Zheng XY, Song XM, Chen G, Zhang T, Wu JX (2008b) Neural tube defects and disturbed maternal folate- and homocysteinemediated one-carbon metabolism. Exp Neurol 212(2):515-521. doi:10.1016/j.expneurol.2008.04.044

Zhang T, Xin R, Gu X, Wang F, Pei L, Lin L, Chen G, Wu J, Zheng X (2009) Maternal serum vitamin B12, folate and homocysteine and the risk of neural tube defects in the offspring in a high-risk area of China. Public Health Nutr 12(5):680-686. doi:10.1017/ S1368980008002735

Zhao H, Wang F, Wang J, Xie H, Guo J, Liu C, Wang L, Lu X, Bao Y, Wang G, Zhong R, Niu B, Zhang T (2012) Maternal PCMT1 gene polymorphisms and the risk of neural tube defects in a Chinese population of Lvliang high-risk area. Gene 505(2):340-344. doi:10.1016/j.gene.2012.05.035

Zhu H, Wicker NJ, Shaw GM, Lammer EJ, Hendricks K, Suarez L, Canfield M, Finnell RH (2003) Homocysteine remethylation enzyme polymorphisms and increased risks for neural tube defects. Mol Genet Metab 78(3):216-221

Zhu H, Yang W, Lu W, Zhang J, Shaw GM, Lammer EJ, Finnell RH (2006) A known functional polymorphism (Ile120Val) of the human PCMT1 gene and risk of spina bifida. Mol Genet Metab 87(1):66-70. doi:10.1016/j.ymgme.2005.09.008 